\title{
A Global Multimoment Constrained Finite-Volume Scheme for Advection Transport on the Hexagonal Geodesic Grid
}

\author{
CHungang CHEN \\ School of Human Settlement and Civil Engineering, Xi'an Jiaotong University, Xi'an, and LHD, \\ Institute of Mechanics, Chinese Academy of Sciences, Beijing, China \\ JUZHONG BIN \\ LHD, Institute of Mechanics, Chinese Academy of Sciences, Beijing, China \\ FENG XIAO \\ Department of Energy Sciences, Tokyo Institute of Technology, Yokohama, Japan
}

(Manuscript received 15 April 2011, in final form 7 August 2011)

\begin{abstract}
A third-order numerical model is developed for global advection transport computation. The multimoment constrained finite-volume scheme has been implemented to the hexagonal geodesic grid for spherical geometry. Two kinds of moments (i.e., point value and volume-integrated average) are used as the constraint conditions to derive the time evolution equations to update the computational variables, which are the values defined at the specified points over each mesh element in the present model. The numerical model has rigorous numerical conservation and third-order accuracy. One of the major merits of the present method is that it does not explicitly involve numerical quadrature, which leads to great convenience in accurately computing curved geometry and source terms. The present paper provides an accurate and practical formulation for advection calculation in the hexagonal-type geodesic grid.
\end{abstract}

\section{Introduction}

Geodesic grids enjoy computational efficiency and overall accuracy from the quasi-global uniformity of mesh spacing, and thus have become increasing popular in the community of global modeling for atmospheric and oceanic dynamics. Some of these models are found in Williamson (1968), Sadourny et al. (1968), Stuhne and Peltier (1999), Tomita et al. (2001), Majewski et al. (2002), Bonaventura and Ringler (2005), Giraldo (2006), Ii and Xiao (2010), and Ringler et al. (2010), among others. Two kinds of control volumes or mesh elements (i.e., triangular Delaunay tessellation and hexagonal Voronoi tessellation), can be straightforwardly generated for constructing finite-volume schemes for the geodesic grids. Since both triangular and hexagonal meshes on the sphere have the

Corresponding author address: Chungang Chen, School of Human Settlement and Civil Engineering, Xi'an Jiaotong University, Xi'an, 710049, China.

E-mail: cgchen@mail.xjtu.edu.cn nature of unstructured grids where the coordinate has to be locally defined and is not continuous at cell boundaries, the conventional high-order reconstructions devised for structured grids cannot be directly applied. The high-order conventional finite-volume method, which uses the cellintegrated value only as the computational variable, usually needs to generate multidimensional interpolation over a cluster of mesh cells, which are not always chosen in the most optimized way, and even worse, choice of the stencil might result in computational instability in some cases for the triangular mesh elements (Friedrich 1998; Weller et al. 2009). Efforts have been so far reported to improve the advection calculation on the hexagonal geodesic grid (Lipscomb and Ringler 2005; Miura 2007; Skamarock and Menchaca 2010). To our knowledge, the existing finite-volume schemes of flux form that make use of the cell-integrated average as the computational variable possess second-order accuracy at most. Our previous studies show that locally increasing the degrees of freedom (DOFs) is a practical and efficient alternative to construct high-order schemes (Ii et al. 2005; Ii and Xiao 
2010; Akoh et al. 2010). Compared to the triangular Delaunay tessellation, the hexagonal Voronoi tessellation is more attractive in global grid uniformity, arrangement of local DOFs for reconstruction, as well as the accuracy and robustness in the flux computation.

In this paper, we present a third-order finite-volume formulation on the hexagonal geodesic grid for global advection transport by using the multimoment constrained finite-volume (MCV) method (Ii and Xiao 2009). Point values at the vertices and the center of each hexagon are used as the computational variables that provide the local DOFs for constructing the piecewise 2D interpolation function. The prognostic equations to update the computational variables are derived from a set of constraint conditions in terms of two kinds of moments (i.e., the values at the vertices are computed from the point-wise derivative Riemann solver and the cell-averaged value is updated from a finite-volume formulation in flux form and is thus rigorously conserved). Given enough DOFs over each hexagonal mesh cell, the predicted point values are only defined at the vertexes unlike the triangular tessellation in Ii and Xiao (2010) where the values at the edge center are also treated as the prognostic variable for a thirdorder scheme.

Section 2 describes the MCV formulation on the hexagonal grid of planar geometry. The implementation on the geodesic hexagonal grid is presented in section 3 . Numerical tests that verify the present scheme are reported in section 4 followed by the conclusions in section 5 .

\section{MCV scheme on planar hexagonal grid}

As shown in Fig. 1, seven local DOFs (denoted by solid circles) [i.e., $\left.\boldsymbol{\phi}_{i_{m}}(m=1,7)\right]$ are defined for a hexagonal element $\mathcal{C}_{i}$, which are located at six vertices from $P_{i_{1}}$ to $P_{i_{6}}$ (counterclockwise) and element center $P_{i_{7}}$. The hexagonal element is compassed by six boundary edges (i.e., $l_{1}$ to $l_{6}$ ). Six auxiliary point values (PVs; denoted by solid triangles) (i.e., $P_{i_{8}}$ to $P_{i_{13}}$ ) are defined at midpoints of boundary edges.

The single-cell-based quadratic polynomial can be constructed based on seven local DOFs within element $\mathcal{C}_{i}$ as

$$
\begin{aligned}
\Phi_{i}(x, y)= & c_{i}^{00}+c_{i}^{10} x+c_{i}^{01} y+c_{i}^{11} x y+c_{i}^{20} x^{2}+c_{i}^{02} y^{2} \\
& +c_{i}^{12}\left(x^{2} y+x y^{2}\right) .
\end{aligned}
$$

The coefficients are determined by solving equation set $\mathbf{A c}=\mathbf{b}$, where $\mathbf{A}$ is a $7 \times 7$ matrix with the $m$ th row vector as

$$
\mathbf{a}_{m}=\left[1, x_{i_{m}}, y_{i_{m}}, x_{i_{m}} y_{i_{m}}, x_{i_{m}}^{2}, y_{i_{m}}^{2}, x_{i_{m}}^{2} y_{i_{m}}+x_{i_{m}} y_{i_{m}}^{2}\right]
$$

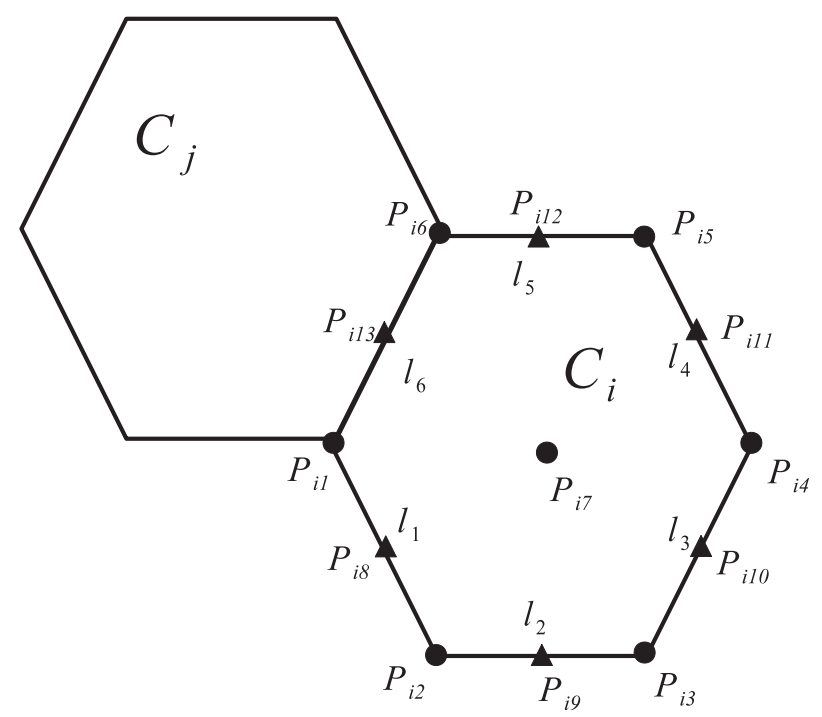

FIG. 1. Configuration of DOFs and constraint conditions in two dimensions for building the third-order MCV scheme.

where $\left(x_{i_{m}}, y_{i_{m}}\right)$ is the location of point $P_{i_{m}}$, vector $\mathbf{c}$ consists of the coefficients in the interpolation function $\Phi_{i}(x, y)$ as

$$
\mathbf{c}=\left[c_{i}^{00}, c_{i}^{10}, c_{i}^{01}, c_{i}^{11}, c_{i}^{20}, c_{i}^{02}, c_{i}^{12}\right]^{\mathrm{T}},
$$

and vector $\mathbf{b}$ consists of DOFs as

$$
\mathbf{b}=\left[\phi_{i_{1}}, \phi_{i_{2}}, \phi_{i_{3}}, \phi_{i_{4}}, \phi_{i_{5}}, \phi_{i_{6}}, \phi_{i_{7}}\right]^{\mathrm{T}} .
$$

Two kinds of moments are adopted as the constraint conditions: the PV moment defined as

$$
\bar{p}_{i_{m}}(t)=\phi\left(x_{i_{m}}, y_{i_{m}}, t\right), \quad(m=1,6)
$$

and the volume-integrated average (VIA) moment defined as

$$
\bar{V}_{i}(t)=\frac{1}{A_{i}} \int_{s} \phi(x, y, t) d s,
$$

where $A_{i}$ is the area of hexagonal element $\mathcal{C}_{i}$.

From (1), the following relation holds between local DOFs and constraint conditions:

$$
\left\{\begin{array}{l}
\phi_{i_{m}}=\bar{P}_{i_{m}} \\
\phi_{i_{7}}=a_{7} \bar{V}_{i}-\sum_{m=1}^{6}\left(a_{m} \bar{P}_{i_{m}}\right)
\end{array} \quad(m=1,6)\right.
$$

where coefficients $a_{1}$ to $a_{7}$ are derived by integrating (1) over the hexagonal element $\mathcal{C}_{i}$. 
Auxiliary PVs defined at midpoints of boundary edges are not independent DOFs. They are evaluated at each time marching step by spatial reconstruction (1) and used in updating PV and VIA constraints.

On planar hexagonal grid, we develop the third-order $\mathrm{MCV}$ scheme for the 2D transport equation:

$$
\partial_{t} \phi+\nabla \cdot \mathbf{f}=0
$$

where $\mathbf{f}=(e, f)=(u \phi, v \phi)$ is the vector of flux function, and $\mathbf{v}=(u, v)$ is the velocity.

Considering the point $P_{l}$ in Fig. 2, we describe the updating procedure for the PV moment defined here. The governing equation of the differential form is adopted, which is written as

$$
\partial_{t}\left(\bar{P}_{l}\right)=-\left(\partial_{x} e\right)_{l}-\left(\partial_{y} f\right)_{l}
$$

The derivatives of fluxes are computed by solving derivative Riemann problem at point $P_{l}$. The formulations used in Ii and Xiao (2009) are adopted as

$$
\begin{aligned}
\left(\partial_{x} e\right)_{l}= & \frac{1}{2}\left[\left(\partial_{x} e\right)_{l}^{-}+\left(\partial_{x} e\right)_{l}^{+}\right] \\
& +\frac{1}{2} \operatorname{SIGN}(1, u)\left[\left(\partial_{x} e\right)_{l}^{-}-\left(\partial_{x} e\right)_{l}^{+}\right]
\end{aligned}
$$

and

$$
\begin{aligned}
\left(\partial_{y} f\right)_{l}= & \frac{1}{2}\left[\left(\partial_{y} f\right)_{l}^{-}+\left(\partial_{y} f\right)_{l}^{+}\right] \\
& +\frac{1}{2} \operatorname{SIGN}(1, v)\left[\left(\partial_{y} f\right)_{l}^{-}-\left(\partial_{y} f\right)_{l}^{+}\right],
\end{aligned}
$$

where superscript "-" denote the left and lower cells and "+" denote right and upper cells, and $\operatorname{SIGN}(a, b)$ returns the absolute value of $a$ and the sign of $b$, which follows the syntax of FORTRAN.

The derivatives of any variable at vertex $P_{l}$ are computed from the local reconstruction over the corresponding cell. For sake of brevity, we consider element $\mathcal{C}_{k}$ in Fig. 2 to give the formulations for evaluating the derivatives. Local coordinates $(\xi, \eta)$ are constructed by choosing two boundary edges of cell $\mathcal{C}_{k}$, which intersect at point $P_{l}$. The derivatives of variable $\psi$ with respect to local coordinates $(\xi, \eta)$ are then evaluated using the point-wise values at points $P_{1}, P_{2}, P_{l}, P_{3}$, and $P_{4}$. In local coordinate system, the locations of these points are $(-1$, $0),(-0.5,0),(0,0),(0,0.5)$, and $(0,1)$, respectively. The derivatives with respect to $\xi$ and $\eta$ are calculated as

$$
\left(\partial_{\xi} \psi\right)_{l}=\psi_{1}-4 \psi_{2}+3 \psi_{l}
$$

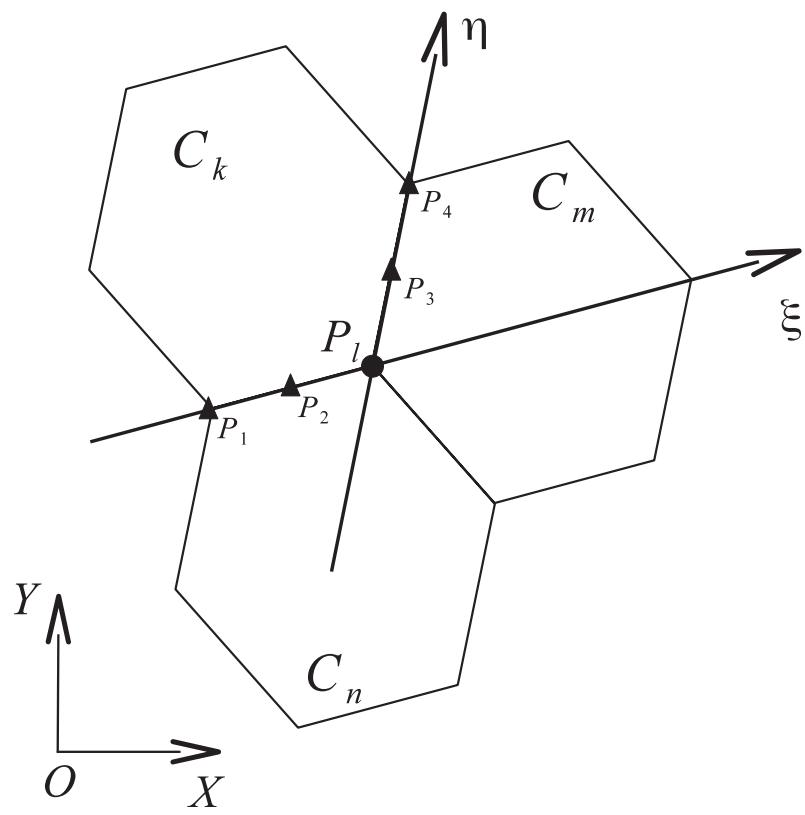

FIG. 2. Updating the PV moment on the hexagonal grid.

and

$$
\left(\partial_{\eta} \psi\right)_{l}=-3 \psi_{l}+4 \psi_{3}-\psi_{4},
$$

where $\psi$ denotes the flux functions $e$ and $f$, as well as the global coordinate $x$ and $y$. Both $\psi_{2}$ and $\psi_{3}$ are the values at the centers of the edges as shown in Fig. 2. For transported field, they are computed by averaging the values from the interpolation functions cell wisely constructed over the two adjacent cells as

$$
\begin{aligned}
& \phi_{2}=\frac{1}{2}\left[\Phi_{k}\left(\mathbf{x}_{2}\right)+\Phi_{n}\left(\mathbf{x}_{2}\right)\right] \quad \text { and } \\
& \phi_{3}=\frac{1}{2}\left[\Phi_{k}\left(\mathbf{x}_{3}\right)+\Phi_{m}\left(\mathbf{x}_{3}\right)\right] .
\end{aligned}
$$

The values of fluxes at centers of edges are then obtained as

$$
\begin{aligned}
& e_{2}=u_{2} \phi_{2}, \quad f_{2}=v_{2} \phi_{2}, \quad e_{3}=u_{3} \phi_{3} \quad \text { and } \\
& f_{3}=v_{3} \phi_{3} .
\end{aligned}
$$

Given the derivatives in local curvilinear coordinates, the derivatives with respect to $x$ and $y$ are obtained through the chain rule as

$$
\begin{aligned}
& \partial_{x} e=\partial_{x} \xi \partial_{\xi} e+\partial_{x} \eta \partial_{\eta} e \\
& \partial_{y} f=\partial_{y} \xi \partial_{\xi} f+\partial_{y} \eta \partial_{\eta} f,
\end{aligned}
$$

where derivatives of $\xi$ and $\eta$ with respect to $x$ and $y$ are obtained by 


$$
\left[\begin{array}{cc}
\partial_{x} \xi & \partial_{x} \eta \\
\partial_{y} \xi & \partial_{y} \eta
\end{array}\right]=\left[\begin{array}{cc}
\partial_{\xi} x & \partial_{\xi} y \\
\partial_{\eta} x & \partial_{\eta} y
\end{array}\right]^{-1}
$$

and the terms in right-hand side are already obtained using (12) and (13).

Integrating transport equation (8) over control volume $\mathcal{C}_{i}$, the flux-form formulation is obtained to update VIA moment as

$$
\partial_{t}\left(\bar{V} \phi_{i}\right)=-\frac{1}{A_{i}} \sum_{m=1}^{6} \int_{l_{m}} \mathbf{f} \cdot \mathbf{n}_{l_{m}} d l
$$

where $l_{m}(m=1,6)$ are boundary edges compassing the control volume $\mathcal{C}_{i}$ as shown in Fig. $1, \mathbf{n}_{l_{m}}$ is the outward normal unit of edge $l_{m}, \mathbf{n}_{l m}=\left(1 /\left|l_{m}\right|\right)\left(y_{i_{m+1}}-y_{i_{m}}, x_{i_{m}}-\right.$ $\left.x_{i_{m+1}}\right)$, and $\left|l_{m}\right|=\sqrt{\left(x_{i_{m+1}}-x_{i_{m}}\right)^{2}+\left(y_{i_{m+1}}-y_{i_{m}}\right)^{2}}$ denotes the length of edge $l_{m}$.

The line integration in (18) is computed by threepoint Simpson's rule as, for example along edge $l_{6}$,

$$
\int_{l_{6}} \mathbf{f} \cdot \mathbf{n}_{l_{6}} d l=\frac{\left|l_{6}\right|}{6}\left(\mathbf{f}_{i_{6}}+\mathbf{f}_{i_{1}}+4 \mathbf{f}_{i_{13}}\right) \cdot \mathbf{n}_{l_{6}},
$$

where $\mathbf{f}_{i_{6}}$ and $\mathbf{f}_{i_{1}}$ are calculated by PVs defined on vertices directly as

$$
\mathbf{f}_{i_{6}}=\left(u_{i_{6}} \phi_{i_{6}}, v_{i_{6}} \phi_{i_{6}}\right), \quad \mathbf{f}_{i_{1}}=\left(u_{i_{1}} \phi_{i_{1}}, v_{i_{1}} \phi_{i_{1}}\right)
$$

and $\mathbf{f}_{i_{13}}$ is obtained by solving Riemann problem in the direction normal to the edge as

$$
\mathbf{f}_{i_{13}}=\frac{v_{n}}{2}\left[\Phi_{i}\left(\mathbf{x}_{i_{13}}\right)+\Phi_{j}\left(\mathbf{x}_{i_{13}}\right)\right]+\frac{\left|v_{n}\right|}{2}\left[\Phi_{i}\left(\mathbf{x}_{i_{13}}\right)-\Phi_{j}\left(\mathbf{x}_{i_{13}}\right)\right],
$$

where $v_{n}=\mathbf{v}_{i_{13}} \cdot \mathbf{n}_{l_{6}}$ is the velocity component in the outward normal direction and $\mathbf{x}_{i_{13}}$ is the location of point $P_{i_{13}}$. The same procedure applies to the line integrations along other edges to get the numerical fluxes required in (18).

Finally, we obtain the evolution equations to update the computational variables (DOFs) by differentiating (7) with respect to time as

$$
\left\{\begin{array}{l}
\partial_{t} \phi_{i_{m}}=\partial_{t}\left(\bar{P}_{i_{i}}\right) \\
\partial_{t} \phi_{i_{7}}=a_{7} \partial_{t}\left(\bar{V}_{i}\right)-\sum_{m=1}^{6}\left[a_{m} \partial_{t}\left(\bar{P}_{i_{m}}\right)\right] .
\end{array} \quad(m=1,6)\right.
$$

The right-hand side of (22) are readily given by the constraints on the PV moment (9) and the VIA moment (18).

To achieve the high-order accuracy in time, the RungeKutta scheme is applied to the semidiscrete evolution equation:

$$
\partial_{t} \phi=\mathcal{L}(\phi),
$$

where $\mathcal{L}$ represents the spatial discretization given in the right-hand side of (22). The third-order total variation diminishing (TVD) Runge-Kutta (Shu 1988) scheme is applied here as

$$
\phi^{n+1}=\phi^{n}+\Delta t\left(K_{1}+K_{2}+4 K_{3}\right),
$$

where

$$
\left\{\begin{array}{l}
K_{1}=\mathcal{L}\left(\phi^{n}\right) \\
K_{2}=\mathcal{L}\left(\phi^{n}+k_{1} \Delta t\right) \\
K_{3}=\mathcal{L}\left(\phi^{n}+\frac{1}{4} k_{1} \Delta t+\frac{1}{4} k_{2} \Delta t\right)
\end{array} .\right.
$$

\section{MCV scheme on hexagonal geodesic grid}

We generate the hexagonal geodesic grid as follows. First, we construct the icosahedral-triangular grid following the method used in Ii and Xiao (2010; see appendix A therein for details). The finer grid is generated by equally partitioning the edge $(L)$ of the 20 primary triangles. We denote a grid whose triangular elements have edge length of $L / n$ by $P=n$. Then the center points of the neighboring five or six triangles sharing the same vertex are connected by great-circle arcs, which form spherical pentagonal or hexagonal elements as shown in Fig. 3. The vertices shared by these triangles now become the center points of the new pentagonal or hexagonal elements on which the MCV scheme is implemented.

The local DOFs are configured in the similar way as the planar case shown in Fig. 1 except that the boundary edges are great-circle arcs in spherical geometry. At the same time, special attention must be paid to the 12 pentagonal elements encompassing the 12 vertices of the 20 primary equilateral triangles. For these elements, we define and use six local DOFs including five at the vertices and one at the cell center.

Spatial reconstruction is implemented in a rotated longitude-latitude coordinates $\left(\lambda^{\prime}, \theta^{\prime}\right)$. The origin of the rotated grid $\left[\left(\lambda^{\prime}, \theta^{\prime}\right)=(0,0)\right]$ is located at the center of the corresponding mesh element. Quadratic polynomials of seven and six DOFs are used for the hexagonal and pentagonal elements, respectively.

In hexagonal elements, the interpolation function $\Phi_{i}\left(\lambda^{\prime}, \theta^{\prime}\right)$ is written as

$$
\begin{aligned}
\Phi_{i}\left(\lambda^{\prime}, \theta^{\prime}\right)= & c_{i}^{00}+c_{i}^{10} \lambda^{\prime}+c_{i}^{01} \theta^{\prime}+c_{i}^{11} \lambda^{\prime} \theta^{\prime} \\
& +c_{i}^{20} \lambda^{\prime 2}+c_{i}^{02} \theta^{\prime 2}+c_{i}^{12}\left(\lambda^{\prime 2} \theta^{\prime}+\lambda^{\prime} \theta^{\prime 2}\right)
\end{aligned}
$$




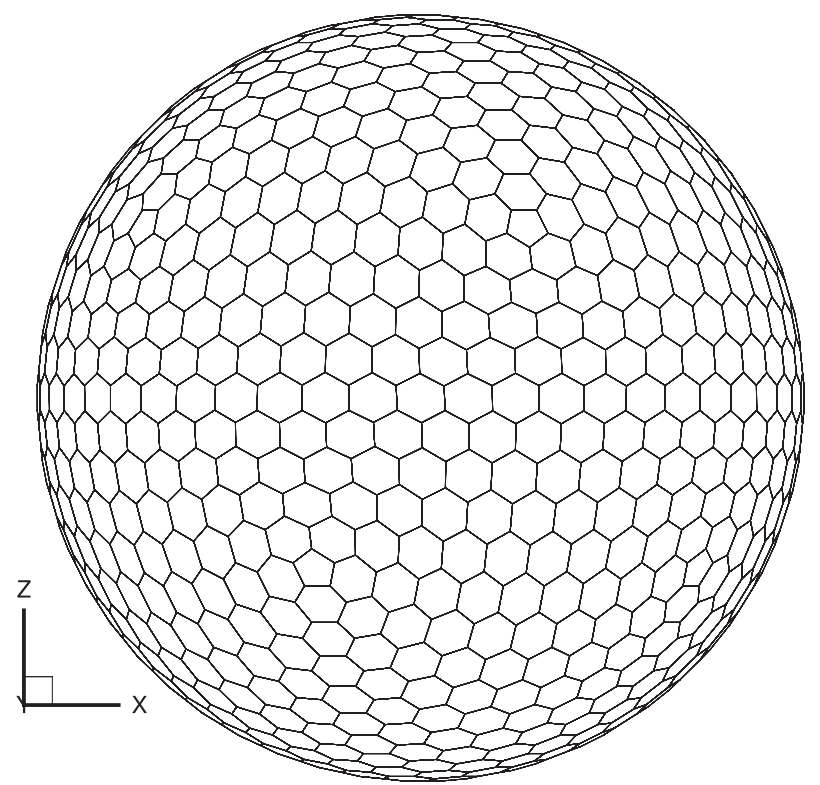

FIG. 3. The spherical icosahedral-hexagonal geodesic grid.

In pentagonal elements, the interpolation function $\Phi_{i}\left(\lambda^{\prime}, \theta^{\prime}\right)$ is written as

$$
\begin{aligned}
\Phi_{i}\left(\lambda^{\prime}, \theta^{\prime}\right)= & c_{i}^{00}+c_{i}^{10} \lambda^{\prime}+c_{i}^{01} \theta^{\prime}+c_{i}^{11} \lambda^{\prime} \theta^{\prime} \\
& +c_{i}^{20} \lambda^{\prime 2}+c_{i}^{02} \theta^{\prime 2} .
\end{aligned}
$$

For mesh element $\mathcal{C}_{i}$ on the sphere surface, constraint conditions are defined by introducing the PV moments at vertices, ${ }^{P} \bar{\phi}_{i}(t)(m=1, M-1)$, and the VIA moment over the mesh element, $\bar{V}_{i}(t)$, where $M$ is the number of local DOFs.

Similar to the planar case, the relation between local DOFs and constraint conditions is

$$
\left\{\begin{array}{l}
\phi_{i_{m}}=\bar{P}_{i_{m}} \\
\phi_{i_{M}}=a_{M} \bar{V}_{i}-\sum_{m=1}^{M-1}\left(a_{m} \bar{P}_{i_{m}}\right)
\end{array} \quad(m=1, M-1)\right.
$$

where coefficients $a_{m}(m=1, M)$ are determined by integrating spatial reconstruction polynomial over the spherical elements. In spherical geometry, the integration is implemented in local curvilinear coordinates. The detailed numerical procedure is described in appendix B.

The PV moments $\bar{P}_{l}$ are updated by the equation of differential form in the 3D Cartesian coordinates $(x, y$, $z$ ) originated at the sphere center:

$\partial_{t}\left(\bar{P}_{l}\right)=-\nabla \cdot \mathbf{f}=-\left(\partial_{x} e\right)_{l}-\left(\partial_{y} f\right)_{l}-\left(\partial_{z} g\right)_{l}$,

where $\mathbf{f}=(e, f, g)=(u \phi, v \phi, w \phi)$ is flux vector, and $(u, v$, $w)$ is the velocity vector in the $3 \mathrm{D}$ Cartesian grid. The

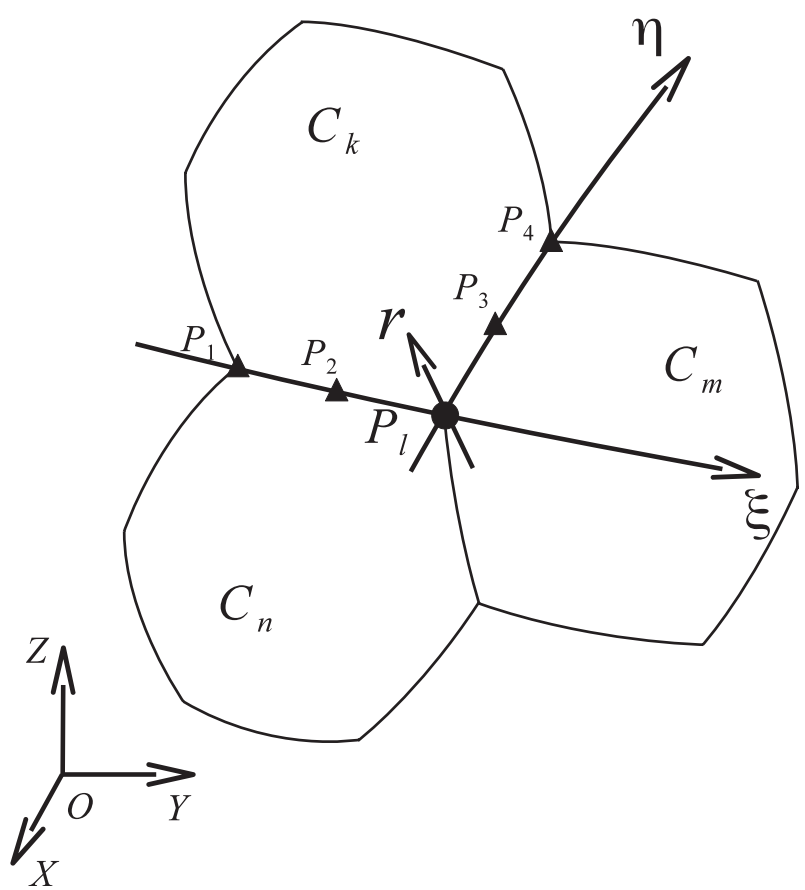

FIG. 4. Local coordinates on the spherical hexagonal grid.

velocity field in the Cartesian grid is connected to that in the longitude-latitude grid $(\tilde{u}, \tilde{v})$ through following relations:

$$
\begin{aligned}
u & =-\tilde{u} \sin \lambda-\tilde{v} \sin \theta \cos \lambda, \\
v & =\tilde{u} \cos \lambda-\tilde{v} \sin \theta \sin \lambda, \\
w & =\tilde{v} \cos \theta
\end{aligned}
$$

Formulations developed in the previous section can be extended to 3D case straightforwardly by including an additional component in the $z$ direction. To update the PV moment, the derivatives of the flux vector are computed by solving derivative Riemann problem. In $x$ and $y$ directions, derivative Riemann problems for flux functions $e$ and $f$ are written exactly as (10) and (11). In the $z$ direction, flux function $g$ is computed as

$$
\begin{aligned}
\left(\partial_{z} g\right)_{l}= & \frac{1}{2}\left[\left(\partial_{z} g\right)_{l}^{-}+\left(\partial_{z} g\right)_{l}^{+}\right] \\
& +\frac{1}{2} \operatorname{SIGN}(1, w)\left[\left(\partial_{z} g\right)_{l}^{-}-\left(\partial_{z} g\right)_{l}^{+}\right] .
\end{aligned}
$$

As shown in Fig. 4, for control volume $\mathcal{C}_{k}$ the local curvilinear coordinate system $(\xi, \eta, r)$ is spanned by two boundary arcs of the computational cell on the sphere surface, $\xi$ and $\eta$, as well as the sphere radius $r$. Given the values of the transported field $(\phi)$, the global coordinates $(x, y, z)$ and the flux function vectors $(e, f, g)$ in 3D Cartesian coordinates at the three points along $\xi$ and $\eta$, respectively, the derivatives with respect to $\xi$ and $\eta$ 


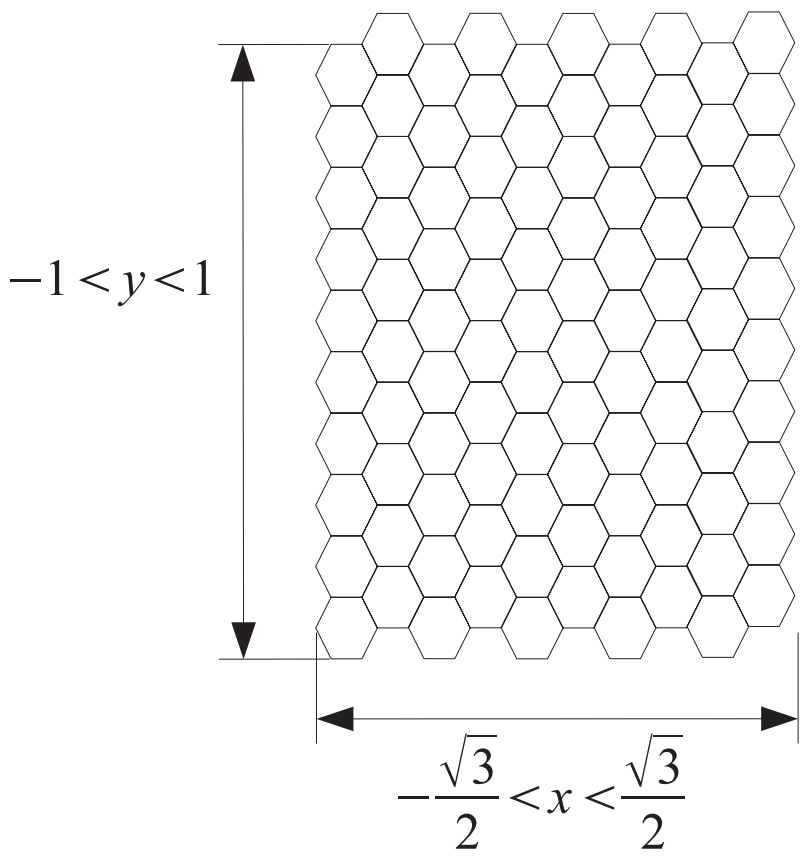

FIG. 5. The planar regular hexagonal grid with a resolution of $10 \times 10$.

are calculated using the three-point formulations (12) and (13) given in the planar case. Consequently, the derivatives of flux functions $e, f$, and $g$ with respect to $x$, $y$, and $z$ are obtained by using the chain rule as

$$
\begin{aligned}
& \partial_{x} e=\partial_{x} \xi \partial_{\xi} e+\partial_{x} \eta \partial_{\eta} e \\
& \partial_{y} f=\partial_{y} \xi \partial_{\xi} f+\partial_{y} \eta \partial_{\eta} f \\
& \partial_{z} g=\partial_{z} \xi \partial_{\xi} g+\partial_{z} \eta \partial_{\eta} g
\end{aligned}
$$

where $\partial_{r} e, \partial_{r} f$, and $\partial_{r} g$ are omitted since the flow is restricted along the surface of sphere.

Similar to the planar case, the metric terms on in righthand side of (32) are obtained as follows:

$$
\left[\begin{array}{lll}
\partial_{x} \xi & \partial_{x} \eta & \partial_{x} r \\
\partial_{y} \xi & \partial_{y} \eta & \partial_{y} r \\
\partial_{z} \xi & \partial_{z} \eta & \partial_{z} r
\end{array}\right]=\left[\begin{array}{ccc}
\partial_{\xi} x & \partial_{\xi} y & \partial_{\xi} z \\
\partial_{\eta} x & \partial_{\eta} y & \partial_{\eta} z \\
\partial_{r} x & \partial_{r} y & \partial_{r} z
\end{array}\right]^{-1}
$$

where $\partial_{r} x, \partial_{r} y$, and $\partial_{r} z$ are analytically given as $x_{l} / R, y_{l} / R$ and $z_{l} / R$ with $R$ being the radius of sphere and $\left(x_{l}, y_{l}, z_{l}\right)$ being the location of point $P_{l}$ in 3D Cartesian grid.

Given the point values of the transported quantity and the velocity field at the vertices and the edge centers, the VIA moment is updated by a flux-form formulation for the mesh element on the sphere surface:

$$
\partial_{t}\left(\bar{V}_{i}\right)=-\frac{1}{A_{i}} \sum_{m=1}^{M-1} \int_{l_{m}} \mathbf{f} \cdot \mathbf{n}_{l_{m}} d l .
$$

When the cell element (Fig. 1) lies on a spherical surface, edge segment $l_{6}$, for example, should be treated as a piece of the great-circle arc. The length of $l_{6}$ is the curved distance between point $P_{i_{6}}=\left(\lambda_{i_{6}}, \theta_{i_{6}}\right)$ and $P_{i_{1}}=\left(\lambda_{i_{1}}, \theta_{i_{1}}\right)$, which is computed by

$\left|l_{6}\right|=R \arccos \left[\sin \theta_{i_{6}} \sin \theta_{i_{1}}+\cos \theta_{i_{6}} \cos \theta_{i_{1}} \cos \left(\lambda_{i_{1}}-\lambda_{i_{6}}\right)\right]$.

The outward normal unit $\mathbf{n}$ of the great-circle $\operatorname{arc} P_{i_{6}} P_{i_{1}}$ is obtained by

$$
\mathbf{n}_{l_{6}}=-\frac{\overrightarrow{O P_{i_{6}}} \times \overrightarrow{O P_{i_{1}}}}{\left|\overrightarrow{O P_{i_{6}}} \times \overrightarrow{O P_{i_{1}}}\right|}
$$

where point $O$ is the origin of Cartesian grid (center of sphere).

Numerical flux across arc $l_{6}$ is computed by

$$
\int_{l_{6}} \mathbf{f} \cdot \mathbf{n}_{l_{6}} d l=\frac{\left|l_{6}\right|}{6}\left(\mathbf{n}_{l_{6}} \cdot \mathbf{v}_{i_{1}} \phi_{i_{1}}+\mathbf{n}_{l_{6}} \cdot \mathbf{v}_{i_{6}} \phi_{i_{6}}+4 \mathbf{n}_{l_{6}} \cdot \mathbf{v}_{i_{13}} \phi_{i_{13}}\right),
$$

where $\phi_{i_{13}}$ is evaluated using the reconstruction polynomial in the upstream element, which is found according to the

\begin{tabular}{|c|c|c|c|c|c|c|}
\hline \multirow[b]{2}{*}{ Grid } & \multicolumn{2}{|c|}{$l_{1}$} & \multicolumn{2}{|c|}{$l_{2}$} & \multicolumn{2}{|c|}{$l_{\infty}$} \\
\hline & Error & Order & Error & Order & Error & Order \\
\hline 10 & $2.6795 \times 10^{-2}$ & - & $2.6802 \times 10^{-2}$ & - & $2.7119 \times 10^{-2}$ & - \\
\hline 20 & $3.0786 \times 10^{-3}$ & 3.12 & $3.0753 \times 10^{-3}$ & 3.12 & $3.1034 \times 10^{-3}$ & 3.13 \\
\hline 40 & $3.7517 \times 10^{-4}$ & 3.04 & $3.7518 \times 10^{-4}$ & 3.04 & $3.7795 \times 10^{-4}$ & 3.04 \\
\hline 80 & $4.6628 \times 10^{-5}$ & 3.01 & $4.6626 \times 10^{-5}$ & 3.01 & $4.6917 \times 10^{-5}$ & 3.01 \\
\hline 160 & $5.8218 \times 10^{-6}$ & 3.00 & $5.8217 \times 10^{-6}$ & 3.00 & $5.8544 \times 10^{-6}$ & 3.00 \\
\hline
\end{tabular}
sign of the velocity component in the outward normal direction $v_{n}=\mathbf{v}_{i_{13}} \cdot \mathbf{n}_{l_{6}}$.

Analogously, the time evolution equations of the DOFs are obtained by differentiating (28) with respect to time $t$ and substituting (29) and (34) to the right-hand

TABLE 1. Convergence test on refining planar regular hexagonal grids. 
TABLE 2. Convergence test on refining spherical grids of the rotating advection in the east direction.

\begin{tabular}{|c|c|c|c|c|c|c|}
\hline \multirow[b]{2}{*}{ Grid } & \multicolumn{2}{|c|}{$l_{1}$} & \multicolumn{2}{|c|}{$l_{2}$} & \multicolumn{2}{|c|}{$l_{\infty}$} \\
\hline & Error & Order & Error & Order & Error & Order \\
\hline 2 & $3.1271 \times 10^{-2}$ & - & $3.0519 \times 10^{-2}$ & - & $3.1976 \times 10^{-2}$ & - \\
\hline 4 & $3.7414 \times 10^{-3}$ & 3.06 & $3.6765 \times 10^{-3}$ & 3.05 & $4.1528 \times 10^{-3}$ & 2.94 \\
\hline 8 & $4.6959 \times 10^{-4}$ & 2.99 & $4.5956 \times 10^{-4}$ & 3.00 & $4.8920 \times 10^{-4}$ & 3.09 \\
\hline 16 & $5.9009 \times 10^{-5}$ & 2.99 & $5.7937 \times 10^{-5}$ & 2.99 & $6.2809 \times 10^{-5}$ & 2.96 \\
\hline 32 & $7.4010 \times 10^{-6}$ & 3.00 & $7.2853 \times 10^{-6}$ & 2.99 & $8.0689 \times 10^{-6}$ & 2.96 \\
\hline
\end{tabular}

sides of the resulting equations. Then the semidiscrete ordinary differential equation (ODE) is solved by the third-order Runge-Kutta scheme shown before.

It is revealed from our previous studies that numerical schemes based on multimoment concepts allow larger Courant-Friedrichs-Lewy (CFL) numbers for stability and are more efficient compared to other numerical methods given the same number of DOFs. Moreover, since there is not any numerical quadrature involved in the $\mathrm{MCV}$ method, it is more attractive not only in computational simplicity and efficiency, but also in accurately dealing with the curved surface.

For real applications, an effective limiter should be devised to remove the spurious oscillations when the transported field has large gradients or discontinuities. An oscillation-less multimoment scheme has been proposed in Akoh et al. (2010) for triangular unstructured mesh. To avoid the spurious oscillations, the derivatives (slopes) at the cell center are introduced as additional constraints, which are not the predicted variables, but calculated from the known moments at each time step in a manner that enforces the monotonicity using the TVD or total variation bounded (TVB) concept. After adjusting the shape of local reconstructions by the slope limiter, the multimoment model can successfully solve the shallowwater equations with strong shocks. In principle, this methodology also applies to the present multimoment model on hexagonal mesh. However, it is more challenging to devise an effective slope limiter for multimoment models on hexagonal mesh, which has more degrees of freedom per element. It seems that the limiting projection based on slope should be of a formulation different from the triangular mesh case. In this paper, we are aiming at investigating the feasibility of developing a high-order model on global hexagonal mesh through multimoment local reconstruction. The design of a limiting mechanism for the proposed model is left as an important future work.

\section{Numerical tests}

In this section, numerical tests are carried out to verify the performance of the proposed MCV global model. The third-order accuracy is confirmed by advection transports of smooth sine wave in both planar and spherical geometries. Furthermore, the cosine bell advection from Williamson's standard test set (Williamson et al. 1992), the moving vortices test case (Nair and Jablonowski 2008), and the divergent flow test case (Nair and Lauritzen 2010) are computed to evaluate the proposed MCV scheme in comparison with other existing global transport models.

To quantitively evaluate the numerical results, we use the following normalized error measurements (Williamson et al. 1992):

$$
\begin{aligned}
& l_{1}=\frac{\int_{S}\left|\phi-\phi_{T}\right| d s}{\int_{S}\left|\phi_{T}\right| d s}, \quad l_{2}=\sqrt{\frac{\int_{S}\left(\phi-\phi_{T}\right)^{2} d s}{\int_{S} \phi_{T}^{2} d s}} \text { and } \\
& l_{\infty}=\frac{\max \left(\left|\phi-\phi_{T}\right|\right)}{\max \left(\left|\phi_{T}\right|\right)}
\end{aligned}
$$

where $S$ is the computational domain and $\phi_{T}$ is the exact solution.

When comparing the present scheme with other global models, we always use the results computed with a similar number of DOFs.

\begin{tabular}{|c|c|c|c|c|c|c|}
\hline \multirow[b]{2}{*}{ Grid } & \multicolumn{2}{|c|}{$l_{1}$} & \multicolumn{2}{|c|}{$l_{2}$} & \multicolumn{2}{|c|}{$l_{\infty}$} \\
\hline & Error & Order & Error & Order & Error & Order \\
\hline 2 & $3.0034 \times 10^{-2}$ & - & $3.0047 \times 10^{-2}$ & - & $3.1911 \times 10^{-2}$ & - \\
\hline 4 & $3.3509 \times 10^{-3}$ & 3.16 & $3.2915 \times 10^{-3}$ & 3.19 & $3.5327 \times 10^{-3}$ & 3.18 \\
\hline 8 & $3.9331 \times 10^{-4}$ & 3.09 & $3.8325 \times 10^{-4}$ & 3.10 & $4.2483 \times 10^{-4}$ & 3.06 \\
\hline 16 & $4.7593 \times 10^{-5}$ & 3.05 & $4.6508 \times 10^{-5}$ & 3.04 & $5.1853 \times 10^{-5}$ & 3.03 \\
\hline 32 & $5.8978 \times 10^{-6}$ & 3.01 & $5.7669 \times 10^{-6}$ & 3.01 & $6.4712 \times 10^{-6}$ & 3.00 \\
\hline
\end{tabular}

TABLE 3. As in Table 2, but for rotating advection in the northeast direction. 
TABLE 4. Normalized errors of the cosine bell advection test on grid $P=36$ in different directions.

\begin{tabular}{cccc}
\hline \hline$\alpha$ & $l_{1}$ & $l_{2}$ & $l_{\infty}$ \\
\hline 0 & $0.3715 \times 10^{-1}$ & $0.2279 \times 10^{-1}$ & $0.1809 \times 10^{-1}$ \\
$\pi / 4$ & $0.3482 \times 10^{-1}$ & $0.2143 \times 10^{-1}$ & $0.1696 \times 10^{-1}$ \\
$\pi / 2$ & $0.3586 \times 10^{-1}$ & $0.2241 \times 10^{-1}$ & $0.1773 \times 10^{-1}$ \\
\hline
\end{tabular}

\section{a. Convergence rate test}

\section{1) Test in two Dimensions}

We calculated the advection of the 2D sine wave on a regular hexagonal grid in planar geometry. A grid with resolution of $10 \times 10$ is shown in Fig. 5. The initial condition is given as

$$
\phi(x, y, 0)=\sin \left[\pi\left(\frac{2 \sqrt{3}}{3} x+y\right)\right]
$$

where $x \in[-\sqrt{3} / 2, \sqrt{3} / 2], y \in[-1,1]$ and $u=\sqrt{3} / 2$, $v=1$.

The normalized errors of tests on refining grids after one complete revolution are shown in Table 1, thirdorder accuracy is obtained as expected.

\section{2) TESTS ON SPHERICAL ICOSAHEDRAL- HEXAGONAL GRID}

A smooth initial condition used for examining the convergence on sphere is designed as

$$
\phi\left(\lambda^{\prime}, \theta^{\prime}\right)=\sin \lambda^{\prime} \cos \theta^{\prime},
$$

where the origin of rotated longitude-latitude coordinates $\left(\lambda^{\prime}, \theta^{\prime}\right)$ is located at $(0, \alpha)$.

The divergence-free velocity field is specified as (Williamson et al. 1992)

$$
\left\{\begin{array}{l}
\tilde{u}=u_{0}(\cos \theta \cos \alpha+\sin \theta \cos \lambda \sin \alpha) \\
\tilde{v}=-u_{0} \sin \lambda \sin \alpha
\end{array},\right.
$$

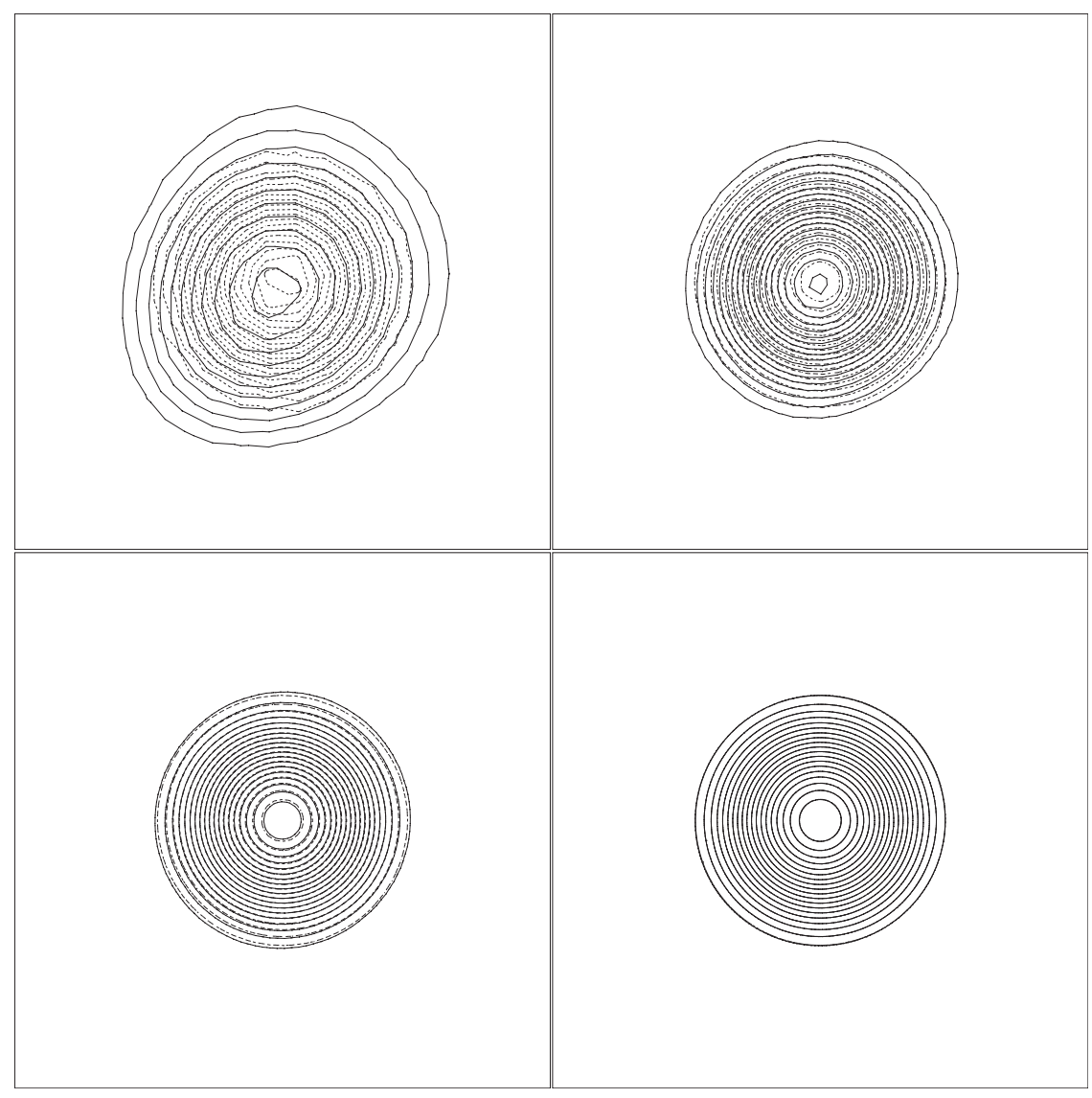

FIG. 6. Numerical results of the cosine bell advection test after running in the northeast direction for one revolution. Shown are height fields (solid) and exact solutions (dashed) on (top left) grid 9, (top right) grid 18, (bottom left) grid 36, and (bottom right) grid 72 . The contour lines vary from 50 to $950 \mathrm{~m}$ by $50 \mathrm{~m}$. 


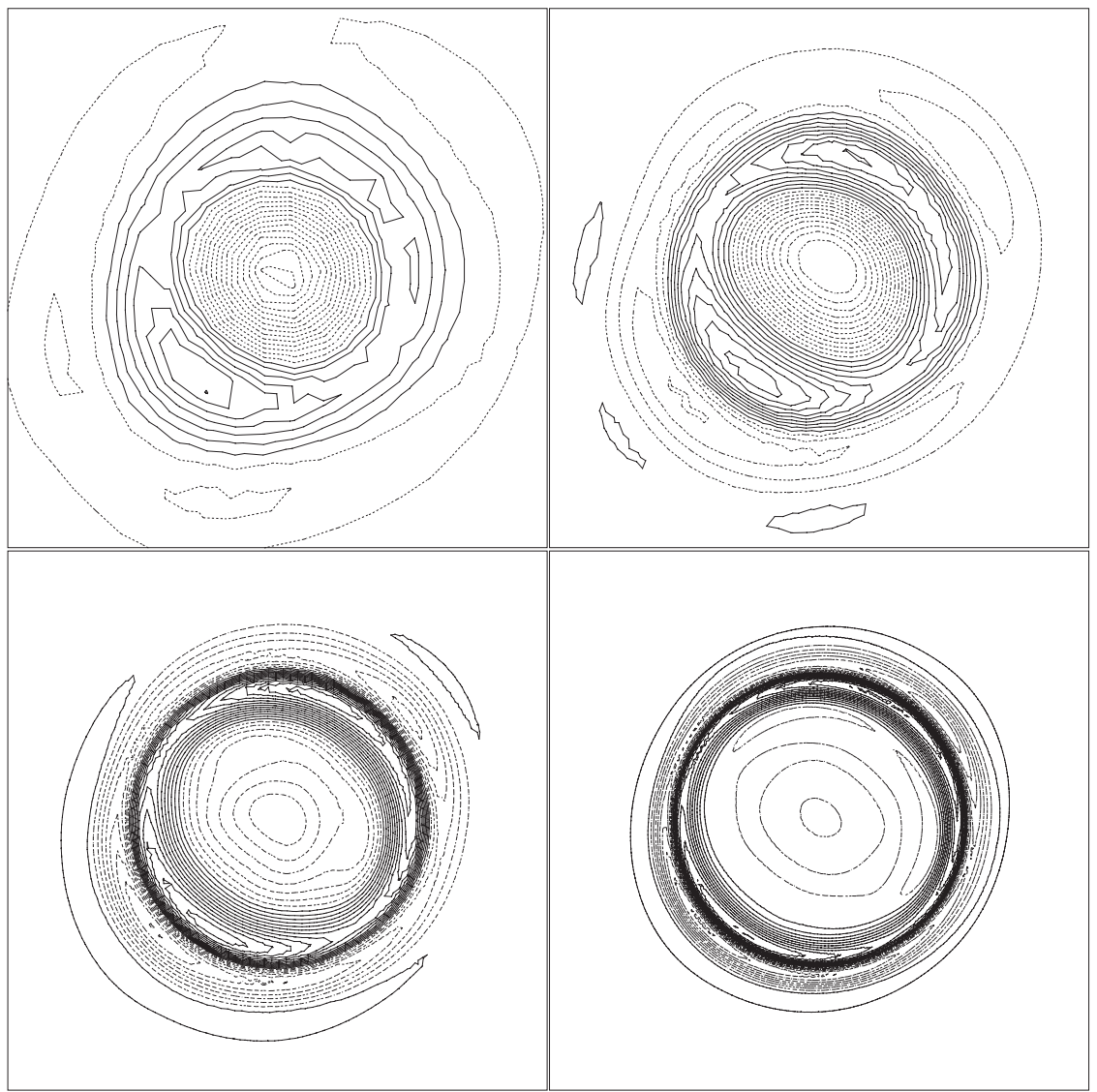

FIG. 7. Numerical results of the cosine bell advection test after running in the northeast direction for one revolution. Shown are absolute errors on (top left) grid 9, (top right) grid 18, (bottom left) grid 36, and (bottom right) grid 72. The contour lines vary from -400 to $200 \mathrm{~m}$ by $30 \mathrm{~m}$ on grid $9,-80$ to $60 \mathrm{~m}$ by $7 \mathrm{~m}$ on grid $18,-10$ to $16 \mathrm{~m}$ by $1.3 \mathrm{~m}$ on grid 36 , and -3.6 to $4.6 \mathrm{~m}$ by $0.41 \mathrm{~m}$ on grid 72 . Negative values are shown by dashed lines.

where $\tilde{u}$ and $\tilde{v}$ are linear velocity in longitude-latitude coordinates, $u_{0}=2 \pi R /(12$ days $)$, and $\alpha$ is the angle between rotation axis and the earth's axis.

Our global model ran on a series of gradually refined grids. Rotations are conducted in east and northeast directions, corresponding to $\alpha=0$, and $\pi / 4$. According to numerical results shown in Tables 2 and 3 , third-order accuracy is achieved in spherical geometry.

\section{b. Cosine bell advection}

As the first case of Williamson's standard test set (Williamson et al. 1992), cosine bell advection is widely tested by many existing models. The initial field is specified as

$\phi(\lambda, \theta, 0)= \begin{cases}\left(\frac{h_{0}}{2}\right)\left[1+\cos \left(\frac{\pi r}{r_{0}}\right)\right] & \text { if } \quad r<r_{0} \\ 0 & \text { otherwise }\end{cases}$ where $r$ is the great-circle distance between any point $(\lambda, \theta)$ and the initial center $(3 \pi / 2,0)$, other constants are specified as $h_{0}=1000 \mathrm{~m}$ and $r_{0}=R / 3$. The divergencefree velocity field (41) is adopted.

The computation is carried out on $P=36$ grid with three rotation directions corresponding to $\alpha=0, \pi / 4$, and $\pi / 2$. A time step of $1440 \mathrm{~s}$ is adopted in the present test. The normalized errors after one revolution are given in Table 4. It is found that the icosahedral-hexagonal global grid eliminates the polar problems and the numerical results are almost independent of flow directions.

This test was calculated by Miura (2007) and Skamarock and Menchaca (2010) on the grids with resolution of levels $4,5,6$, and 7, where total numbers of DOFs are 2562 , 10 242, 40 962, and 163 842, respectively. To compare with their results, we run this test on refined global grids with $P=9,18,36$, and 72. Corresponding numbers of DOFs are 2432, 9722, 38 882, and 155 522, which are a little less than their calculations. Numerical results of the transported field and absolute errors after rotating one revolution in 


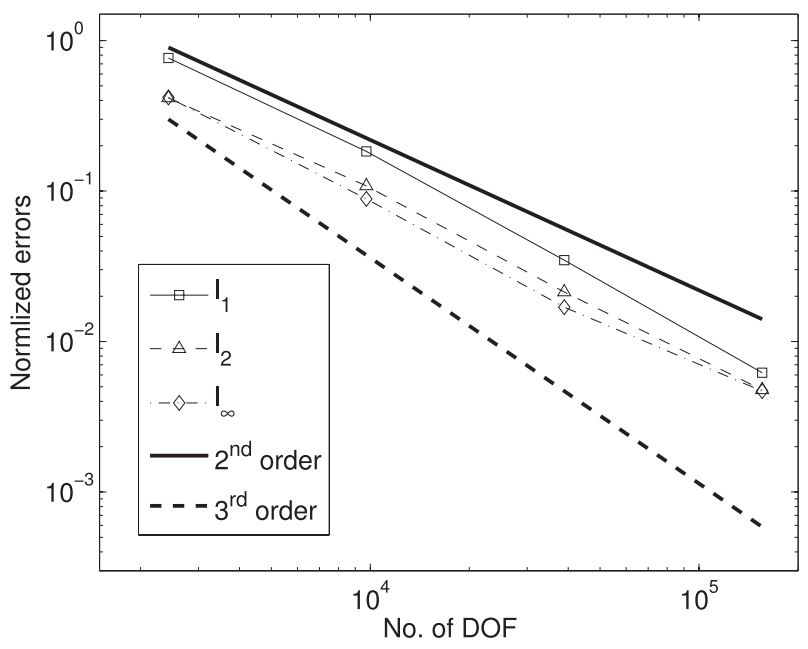

FIG. 8. Normalized errors of the cosine bell advection in the northeast direction on refining grids.

the northeast direction on different grids are shown in Figs. 6 and 7. Distortion and phase error are observed on the very coarse grid $P=9$. With the increase of grid resolution, the numerical accuracy for shape and phase is improved significantly. On the finest $\operatorname{grid} P=72$, there is hardly a visible difference between the numerical result and the exact solution. Normalized errors on different grids are shown in Fig. 8. Because of the discontinuity along the boundary of cosine bell, the convergence rate of present scheme is about second order. Our results are better than those given in Miura (2007) without monotone limiter (see their Fig. 4b). Compared to the recent scheme of Skamarock and Menchaca (2010), which uses secondorder reconstruction and is more accurate than those in Miura (2007), our model gives smaller $l_{\infty}$ errors, but larger $l_{2}$ errors. In Skamarock and Menchaca (2010), the influence
TABLE 5. Normalized errors of the moving vortex test on grid $P=$ 18 in different directions.

\begin{tabular}{cccc}
\hline \hline$\alpha$ & $l_{1}$ & $l_{2}$ & $l_{\infty}$ \\
\hline 0 & $0.9138 \times 10^{-2}$ & $0.2531 \times 10^{-1}$ & 0.1066 \\
$\pi / 4$ & $0.8799 \times 10^{-2}$ & $0.2486 \times 10^{-1}$ & 0.1107 \\
$\pi / 2$ & $0.8810 \times 10^{-2}$ & $0.2487 \times 10^{-1}$ & 0.1065 \\
\hline
\end{tabular}

of time step is studied. For the present scheme, however, our numerical experiments show that the normalized errors are hardly affected by the time step.

A third-order MCV scheme is devised in Ii and Xiao (2010) on the icosahedral-triangular grid. They ran this test on a grid with $37502 \mathrm{DOFs}$, almost equivalent to a $P=36$ grid in terms of DOFs. Numerical results show that the third-order MCV scheme on hexagonal tessellation produces less numerical error than triangular tessellation given the same number of DOFs.

\section{c. Moving vortices test}

To check our model with a more realistic test case, we computed the moving vortices test recently proposed in Nair and Jablonowski (2008). In this test, the transported field deforms around two vortex centers. Meanwhile, the vortex centers travel along the trajectory of the previous solid rotation test. Detailed configuration of this test is found in Nair and Jablonowski (2008). In this study, we define the initial condition with the vortex center located at $(3 \pi / 2,0)$. Three rotation directions are tested, corresponding to $\alpha=0$, $\pi / 4$, and $\pi / 2$.

This test is checked on three refined grids: $P=9, P=$ 18 , and $P=36$. Total numbers of DOFs on these three grids are similar to longitude-latitude grids with resolutions of $5^{\circ}, 2.5^{\circ}$, and $1.25^{\circ}$. The MCV model ran on
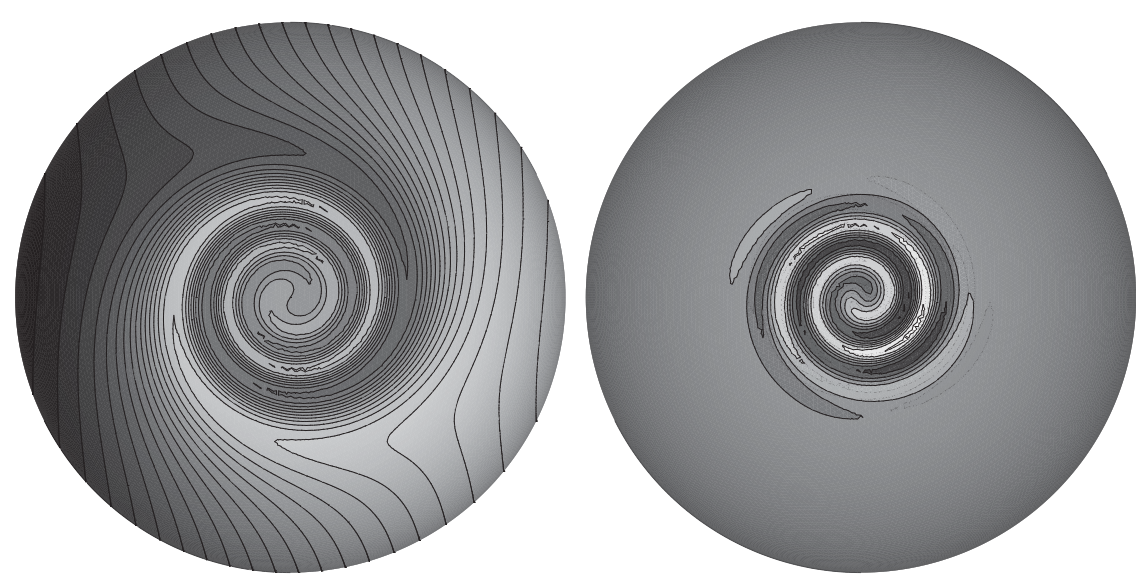

FIG. 9. Numerical results of the moving vortices' test on grid $P=36$ at day 12. (left) The height field (contour lines from 0.5 to 1.5 by 0.05 ) and (right) the absolute error (contour lines from -0.07 to 0.07 by 0.02 ). 

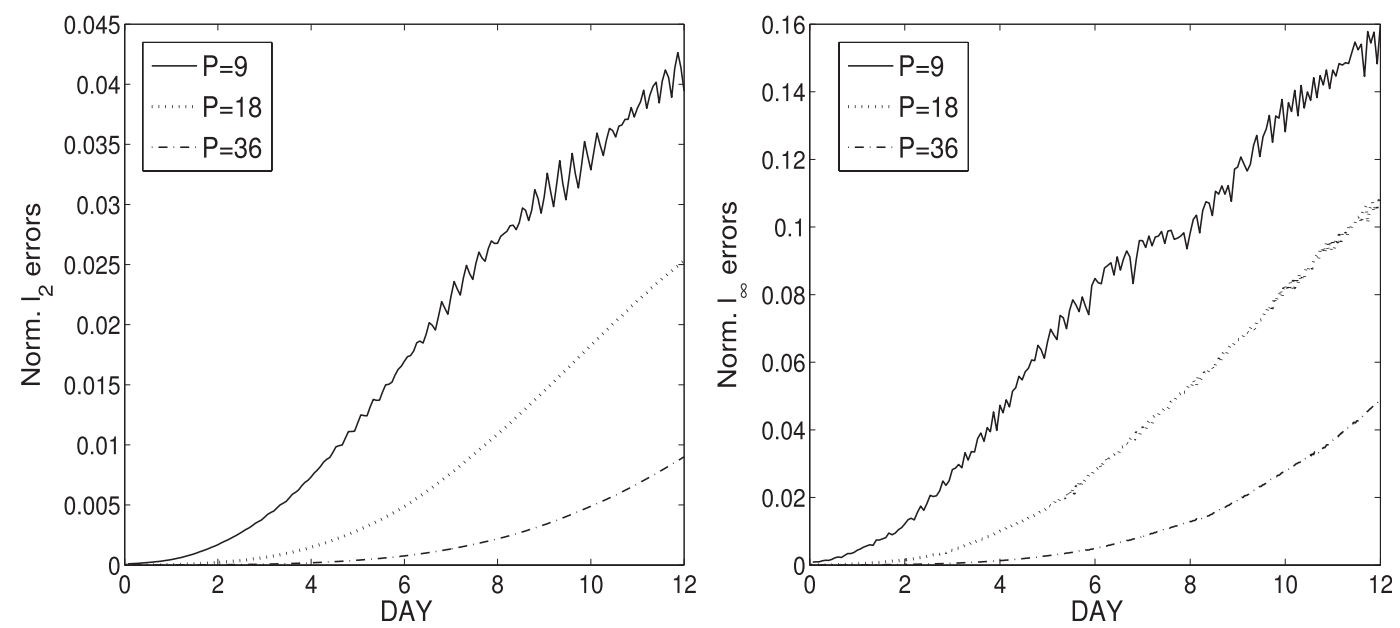

FIG. 10. Normalized errors of the moving vortices' test on a different grid. (left) Normalized $l_{2}$ errors and (right) $l_{\infty}$ errors.

$P=18$ grid to day 12 (one complete revolution for vortex center to return to its original location) in different directions and the normalized errors are given in Table 5. Numerical results on the spherical icosahedralhexagonal grid are almost independent of rotation directions. The numerical result and absolute error of the transported field on $P=36$ grid with $\alpha=0$ are given in Fig. 9.

Time history of normalized $l_{2}$ and $l_{\infty}$ errors are shown in Fig. 10. The present model gives competitive results compared to the finite-volume solution in Nair and Jablonowski (2008, see their Fig. 7).

Compared with the third-order MCV model on the icosahedral-triangular grid (Ii and Xiao 2010), the present model appears to be more accurate. The present model with 38882 DOFs $(P=36)$ produces results comparable to those from the icosahedral-triangular grid with DOFs of 54002 [see Fig. 9 in Ii and Xiao (2010) for the normalized errors].

\section{d. Divergent flow test}

A divergent flow test was proposed in Nair and Lauritzen (2010). On a unit sphere, the flow field is given as

$$
\left\{\begin{array}{l}
\tilde{u}(\lambda, \theta, t)=-\sin ^{2}\left(\frac{\lambda}{2}\right) \sin (2 \theta) \cos ^{2} \theta \cos \left(\frac{\pi t}{5}\right) \\
\tilde{v}(\lambda, \theta, t)=\frac{1}{2} \sin \lambda \cos ^{3} \theta \cos \left(\frac{\pi t}{5}\right)
\end{array} .\right.
$$

The initial fluid density is specified as $\rho=1$ and initial tracer concentration is

$$
\phi= \begin{cases}b+c h_{1}(\lambda, \theta) & \text { if } r_{1}<r_{0} \\ b+c h_{2}(\lambda, \theta) & \text { if } r_{2}<r_{0} \\ b & \text { otherwise }\end{cases}
$$

where $b=0.1, c=0.9, h_{1}$, and $h_{2}$ are two cosine bells given in (42) with centers at $(3 \pi / 4,0)$ and $(5 \pi / 4,0)$, other parameters are $h_{0}=1$ and $r_{0}=1 / 2$.

We solve the flux-form governing equation [Eq. (2) in Nair and Lauritzen (2010)] to predict the evolution of tracer density directly. In this test, the tracer density deforms from circular pattern of cosine bell into a narrow stretched band during the first half period of computation. At half-time $t=T / 2$, tracer density field reaches the extreme deformation. Then the inverse velocity is applied and finally the tracer density field will return to its initial shape at $t=T$. This test is checked on the $P=36$ grid by the third-order MCV model. Numerical results are shown in Fig. 11 for tracer density at $t=0,2.5(T / 2)$ and $5(T)$. At $t=T / 2$, the tracer density field is visibly identical to the reference solution by the Discontinuous Galerkin (DG) model (see Fig. 3 in Nair and Lauritzen 2010). After one revolution, the shape and phase of two cosine bells are preserved well compared with initial condition. Normalized errors for this test are $l_{1}=0.8245 \times 10^{-2}, l_{2}=0.1768$ $\times 10^{-1}$, and $l_{\infty}=0.3089 \times 10^{-1}$. It is found that the $l_{2}$ error is comparable to solid rotation of the cosine bell (shown in Table 4) on the same grid. Similar results are found in the DG solutions of different test cases [see Fig. 3 in Nair et al. (2005) for the solid rotation case and Table 1 in Nair and Lauritzen (2010) for the divergent flow case]. The present model can accurately deal with the divergent flow field.

\section{Conclusions}

We present a third-order advection transport scheme in spherical geometry by implementing the multimoment 

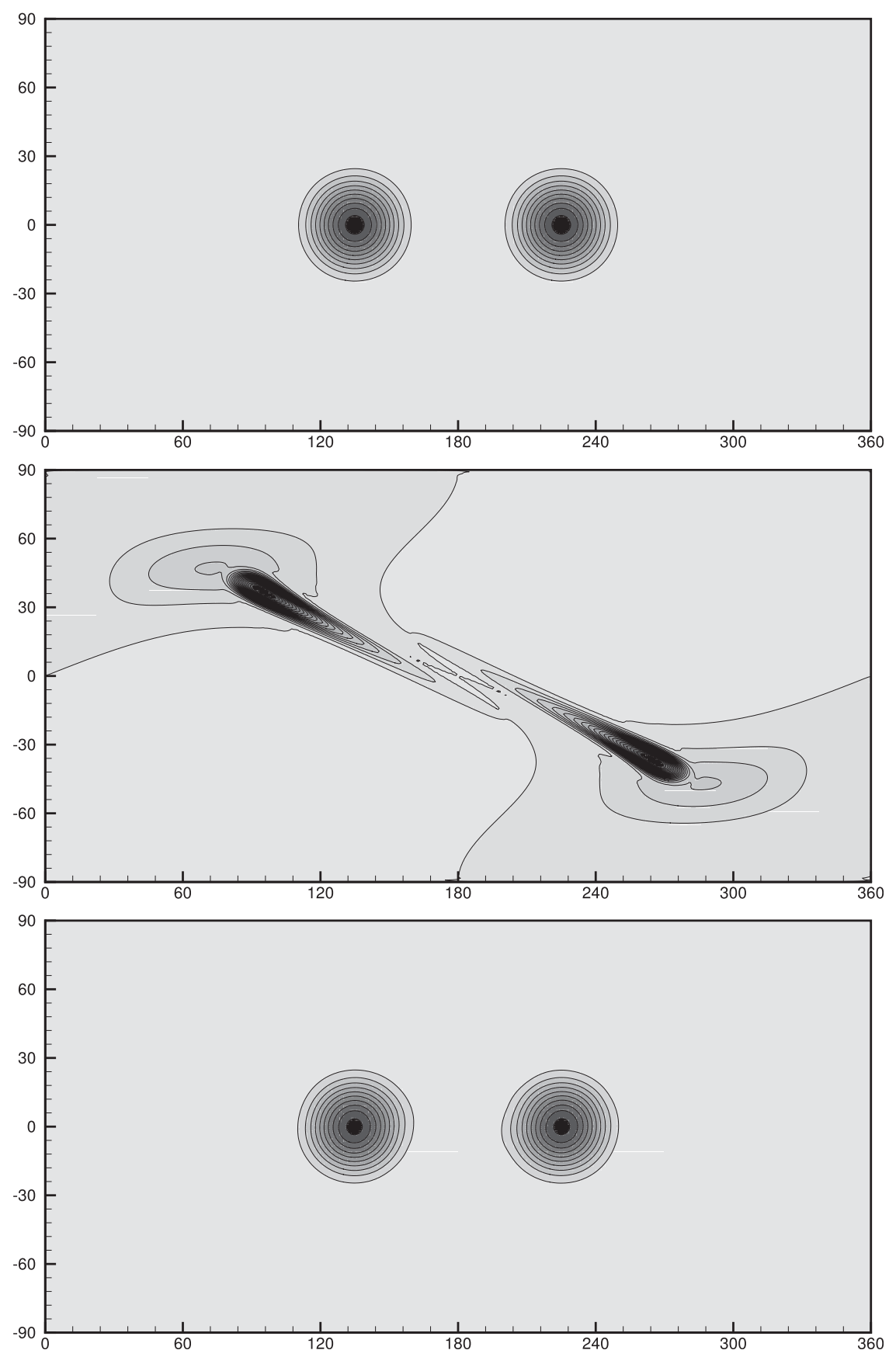

FIG. 11. Numerical results of the tracer density of the divergent flow test. Tracer density at (top) $t=0$, (middle) $t=2.5$, and (bottom) $t=5$.

constrained finite-volume method to the hexagonal geodesic grid. The cell-wisely constructed interpolation is well suited for the unstructured mesh configuration and gives a uniform third-order accuracy in various numerical tests. Compared to the conventional finite-volume method that requires a wide stencil for constructing a high-order scheme, the present scheme uses locally defined DOFs and has a significant advantage when applied to geodesic grids.

Our previous studies (Ii and Xiao 2010) show that the $\mathrm{MCV}$ formulation is also promising for dynamic cores. As a matter of fact, we have developed a shallow-water model under the same MCV framework for the hexagonaltype geodesic grid, which will be separately reported soon. The advection transport model presented in this 
paper can be embedded straightforwardly as a part of such a model. It is noted that the present model can also be used as an independent module to plug in to models based on other traditional finite-volume formulations if a scheme is devised to communicate the values between the cell center and the vertices.

Acknowledgments. This work is supported by the National Natural Science Foundation of China (Project 10902116) and the Chinese Academy of Sciences (Project KJCX2-YW-L04).

\section{APPENDIX A}

\section{Accuracy Analysis on VIA Moment}

In this section, we prove the third-order accuracy of the spatial discretization formulation for the VIA moment. Shown in Fig. A1, we consider the VIA moment $\bar{V}_{i}$ over control volume $\mathcal{C}_{i}$. The transport with constant velocity $u, v=1$ on the planar regular hexagonal element is analyzed. Considering the transported field $\phi$ $(x, y)$ with enough smoothness, we express its Taylor expansion in respect to the cell center as

$$
\begin{aligned}
\phi_{T}(x, y)= & \phi_{7}+\left(\partial_{x} \phi\right)_{7} \tilde{x}+\left(\partial_{y} \phi\right)_{7} \tilde{y}+\frac{1}{2}\left(\partial_{x x} \phi\right)_{7} \tilde{x}^{2}+\frac{1}{2}\left(\partial_{y y} \phi\right)_{7} \tilde{y}^{2} \\
& +\left(\partial_{x y} \phi\right)_{7} \tilde{x} \tilde{y}+\frac{1}{6}\left(\partial_{x x x} \phi\right)_{7} \tilde{x}^{3}+\frac{1}{6}\left(\partial_{y y y} \phi\right)_{7} \tilde{y}^{3}+\frac{1}{2}\left(\partial_{x x y} \phi\right)_{7} \tilde{x}^{2} \tilde{y} \\
& +\frac{1}{2}\left(\partial_{x y y} \phi\right)_{7} \tilde{x} \tilde{y}^{2}+\frac{1}{24}\left(\partial_{x x x x} \phi\right)_{7} \tilde{x}^{4}+\frac{1}{24}\left(\partial_{y y y y} \phi\right)_{7} \tilde{y}^{4}+\frac{1}{4}\left(\partial_{x x y y} \phi\right)_{7} \tilde{x}^{2} \tilde{y}^{2} \\
& +\frac{1}{6}\left(\partial_{x x x y} \phi\right)_{7} \tilde{x}^{3} \tilde{y}+\frac{1}{6}\left(\partial_{x y y y} \phi\right)_{7} \tilde{x} \tilde{y}^{3}+\mathcal{O}\left(L^{5}\right),
\end{aligned}
$$

where $\tilde{x}=x-x_{7}, \tilde{y}=y-y_{7}$, and $L$ is the length of the edge of the hexagonal element.

The flux function is then computed as $\mathbf{f}_{T}=\mathbf{v} \phi(x, y)=$ $\mathbf{i} \phi_{T}(x, y)+\mathbf{j} \phi_{T}(x, y)$. When an analytical integration of the flux function along the boundary edges is applied, the net flux across the boundary of element $\mathcal{C}_{i}$ reads as

$$
\begin{aligned}
\Delta \mathbf{f}_{T}= & \frac{3 \sqrt{3}}{2} L^{2}\left[\left(\partial_{x} \phi\right)_{7}+\left(\partial_{y} \phi\right)_{7}\right]+\frac{5 \sqrt{3}}{32} L^{4}\left[\left(\partial_{x x x} \phi\right)_{7}\right. \\
& \left.+\left(\partial_{y y y} \phi\right)_{7}+\left(\partial_{x x y} \phi\right)_{7}+\left(\partial_{x y y} \phi\right)_{7}\right]+\mathcal{O}\left(L^{6}\right) .
\end{aligned}
$$

We show next that the formulation of the numerical flux in our model results in an approximation coincidence with the leading terms of the exact net flux given in (A2).

In the present scheme, the numerical flux is computed by the three-point Simpson's rule, where the values at the middle points of boundary edges are interpolated from the local seven-point reconstructions of the upstream elements. For the given velocity, the value of the transported field at point $P_{21}$ is computed from the interpolation function on $\mathcal{C}_{m}$, and analogously $P_{22}$ from $\mathcal{C}_{n}, P_{23} \sim P_{25}$ from $\mathcal{C}_{i}$, and $P_{26}$ from $\mathcal{C}_{l}$. All these middle point values, as well as the numerical net flux, are then expressed in terms of the point values at $P_{1}$ to $P_{20}$ (i.e., the predicted variables in the present scheme). The net numerical flux finally reads as

$$
\begin{aligned}
\Delta \mathbf{f}_{N}= & L\left[\left(-\frac{1}{12}-\frac{5}{3 \sqrt{3}}\right) \phi_{1}+\left(-\frac{2}{3}-\frac{43}{48 \sqrt{3}}\right) \phi_{2}+\left(-\frac{19}{24}+\frac{2}{3 \sqrt{3}}\right) \phi_{3}+\left(-\frac{1}{12}+\frac{13}{8 \sqrt{3}}\right) \phi_{4}+\left(\frac{19}{24}+\frac{1}{2 \sqrt{3}}\right) \phi_{5}\right. \\
& +\left(\frac{2}{3}-\frac{35}{48 \sqrt{3}}\right) \phi_{6}+\left(\frac{1}{6}+\frac{1}{2 \sqrt{3}}\right) \phi_{7}+\frac{5}{48 \sqrt{3}} \phi_{8}+\left(-\frac{1}{24}+\frac{1}{48 \sqrt{3}}\right) \phi_{9}+\left(\frac{1}{24}-\frac{1}{48 \sqrt{3}}\right) \phi_{10}+\frac{7}{24 \sqrt{3}} \phi_{11} \\
& +\left(\frac{1}{12}-\frac{1}{4 \sqrt{3}}\right) \phi_{12}+\left(-\frac{1}{24}-\frac{1}{48 \sqrt{3}}\right) \phi_{13}+\left(\frac{1}{24}+\frac{1}{48 \sqrt{3}}\right) \phi_{14}+\left(\frac{1}{12}+\frac{13}{48 \sqrt{3}}\right) \phi_{15}+\left(-\frac{1}{12}-\frac{1}{4 \sqrt{3}}\right) \phi_{16} \\
& \left.-\frac{1}{6 \sqrt{3}} \phi_{17}+\frac{1}{6 \sqrt{3}} \phi_{18}+\left(\frac{1}{12}+\frac{1}{6 \sqrt{3}}\right) \phi_{19}+\frac{1}{6} \phi_{20}\right] .
\end{aligned}
$$




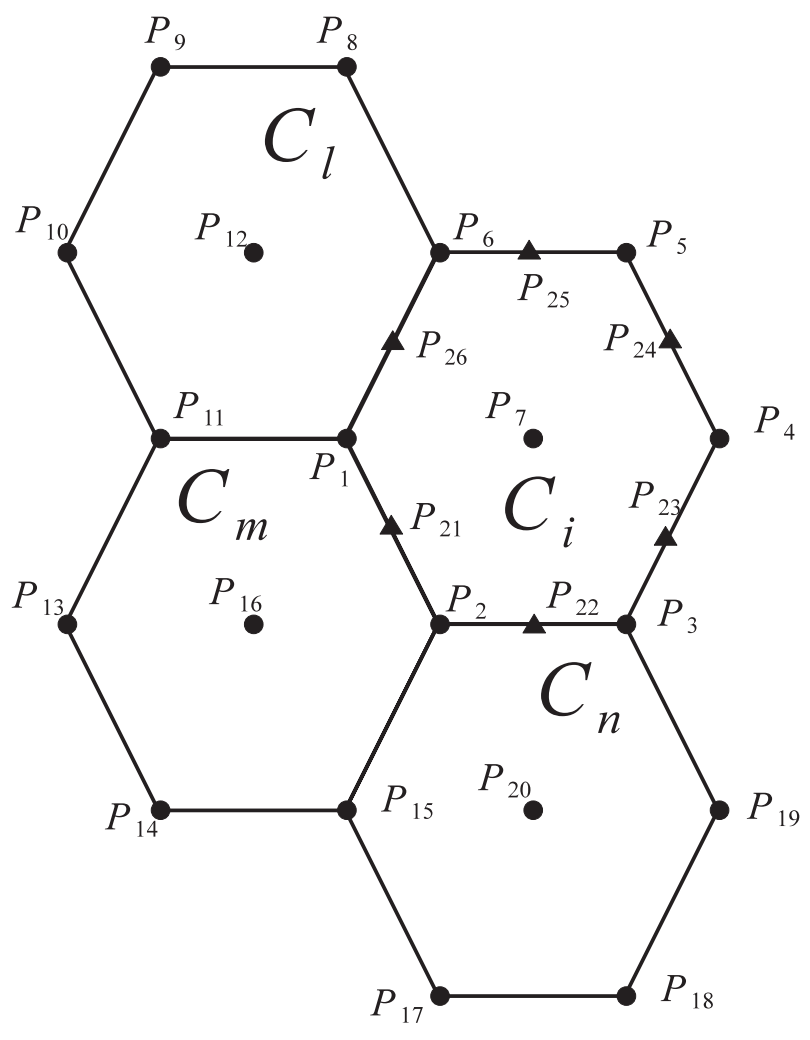

FIG. A1. Control volumes for accuracy analysis on the VIA moment.

Substitute $\phi_{i}=\phi_{T}\left(x_{i}, y_{i}\right),(i=1$ to 20$)$, into (A3), the numerical net flux is recast into

$$
\begin{aligned}
\Delta \mathbf{f}_{N}= & \frac{3 \sqrt{3}}{2} L^{2}\left[\left(\partial_{x} \phi\right)_{7}+\left(\partial_{y} \phi\right)_{7}\right]+\frac{5 \sqrt{3}}{32} L^{4}\left[\left(\partial_{x x x} \phi\right)_{7}\right. \\
& \left.+\left(\partial_{y y y} \phi\right)_{7}+\left(\partial_{x x y} \phi\right)_{7}+\left(\partial_{x y y} \phi\right)_{7}\right]+\mathcal{O}\left(L^{5}\right),
\end{aligned}
$$

which reveals that the net numerical flux in the present scheme results in a fifth-order truncation error.

From the constraint to update the VIA moment (18) and recall the area of the regular hexagonal cell $\mathcal{C}_{i}, A_{i}=$ $(3 / 2) L^{2}$, we arrive at

$$
\partial_{t}\left(\bar{V}_{i}\right)=-\frac{1}{A_{i}} \Delta \mathbf{f}_{N}+\mathcal{O}\left(L^{3}\right)
$$

\section{APPENDIX B}

\section{Surface Integration over the Spherical-Hexagonal Element}

Shown in Fig. B1, the hexagonal element $\mathcal{C}_{i}$ is first divided into six subtriangles, denoted by $\mathcal{C}_{i_{m}}(m=1,6)$, by

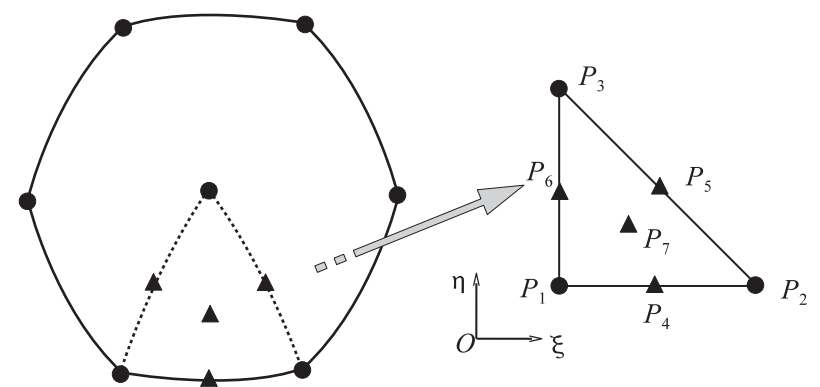

FIG. B1. Surface integration over the spherical hexagonal element.

simply connecting the vertices and centroid. Then the surface integration over a spherical-hexagonal element can be computed with the formulations derived in Ii and Xiao (2010) for the spherical triangles. To maintain highorder accuracy, we sample seven points for each triangular area $\left(\mathcal{C}_{i_{m}}\right)$ (i.e., three vertices, three midpoints of boundary edges, and the centroid of the triangle). The values at the three midpoints of the boundary edges and the centroid are computed from the reconstruction polynomial (26). The surface-integrated average of the transported field $\phi$ over triangle $\mathcal{C}_{i_{m}}$ can be obtained in the local coordinate as

$$
\bar{V}_{i_{m}}=\frac{1}{A_{i_{m}}} \sum_{n=1}^{7}\left(\alpha_{n}|J|_{n} \phi_{n}\right),
$$

where

$\alpha_{1}, \alpha_{2}, \alpha_{3}=\frac{1}{40}, \quad \alpha_{4}, \alpha_{5}, \alpha_{6}=\frac{1}{15}, \quad \alpha_{7}=\frac{9}{40}$,

and $|J|_{n}$ is the point-wisely defined Jacobian of the transformation, which is computed by

$$
|J|=R^{2} \cos \theta\left|\lambda_{\xi} \theta_{\eta}-\lambda_{\eta} \theta_{\xi}\right|
$$

The area of the spherical triangle is

$$
A_{i_{m}}=\sum_{n=1}^{7}\left(\alpha_{n}|J|_{n}\right) .
$$

Then the surface-integrated average of the transported field over the hexagonal element can be obtained by summing up those of the six triangular areas as

$$
{\overline{{ }^{\prime}}}_{i}=\frac{\sum_{m=1}^{6}\left(A_{i_{m} \bar{V}_{i_{m}}}\right)}{\sum_{m=1}^{6} A_{i_{m}}} .
$$


Surface integration over the pentagonal elements is conducted using the same method, except that the element is divided into five triangular areas.

\section{REFERENCES}

Akoh, R., S. Ii, and F. Xiao, 2010: A multi-moment finite volume formulation for shallow water equations on unstructured mesh. J. Comput. Phys., 229, 4567-4590.

Bonaventura, L., and T. Ringler, 2005: Analysis of discrete shallowwater models on geodesic Delaunay grids with C-type staggering. Mon. Wea. Rev., 133, 2351-2373.

Friedrich, O., 1998: Weighted essentially non-oscillatory schemes for the interpolation of mean values on unstructured grids. J. Comput. Phys., 144, 194-212.

Giraldo, F., 2006: High-order triangle-based discontinuous Galerkin methods for hyperbolic equations on a rotating sphere. J. Comput. Phys., 214, 447-465.

Ii, S., and F. Xiao, 2009: High order multi-moment constrained finite volume method. Part I: Basic formulation. J. Comput. Phys., 228, 3669-3707.

— , and —, 2010: A global shallow water model using high order multi-moment constrained finite volume method and icosahedral grid. J. Comput. Phys., 229, 1774-1796.

_, M. Shimuta, and F. Xiao, 2005: A 4th-order and single-cellbased advection scheme on unstructured grids using multimoments. Comput. Phys. Commun., 173, 17-33.

Lipscomb, W., and T. Ringler, 2005: An incremental remapping transport scheme on a spherical geodesic grid. Mon. Wea. Rev., 133, 2235-2250.

Majewski, D., D. Liermann, P. Prohl, B. Ritter, M. Buchhold, T. Hanisch, G. Paul, and W. Wergen, 2002: The operational global icosahedral-hexagonal grid-point model GME: Description and high-resolution tests. Mon. Wea. Rev., 130, 319-338.

Miura, H., 2007: An upwind-biased conservative advection scheme for spherical hexagonal-pentagonal grids. Mon. Wea. Rev., 135, 4038-4044.
Nair, R. D., and C. Jablonowski, 2008: Moving vortices on the sphere: A test case for horizontal advection problems. Mon. Wea. Rev., 136, 699-711.

— , and P. H. Lauritzen, 2010: A class of deformational flow test cases for linear transport problems on the sphere. J. Comput. Phys., 229 (23), 8868-8887.

- S. J. Stephen, and R. D. Loft, 2005: A discontinuous Galerkin transport scheme on the cubed sphere. Mon. Wea. Rev., 133, 814-828.

Ringler, T., J. Thuburn, J. Klemp, and W. Skamarock, 2010: A unified approach to energy conservation and potential vorticity dynamics on arbitrarily structured C-grids. J. Comput. Phys., 229, 3065-3090.

Sadourny, R., A. Arakawa, and Y. Mintz, 1968: Integration of the nondivergent barotropic vorticity equation with an icosahedralhexagonal grid for the sphere. Mon. Wea. Rev., 96, 351-356.

Shu, C. W., 1988: Total variation diminishing time discretization. SIAM J. Sci. Stat. Comput., 9, 1073-1084.

Skamarock, W. C., and M. Menchaca, 2010: Conservative transport schemes for spherical geodesic grids: High-order reconstructions for forward-in-time schemes. Mon. Wea. Rev., 138, $4497-4508$.

Stuhne, G., and W. Peltier, 1999: New icosahedral grid-point discretizations of the shallow water equations on the sphere. J. Comput. Phys., 148, 23-58.

Tomita, H., M. Tshugawa, M. Satoh, and K. Goto, 2001: Shallow water model on a modified icosahedral geodesic grid by using spring dynamics. J. Comput. Phys., 174, 579-613.

Weller, H., H. G. Weller, and A. Fournier, 2009: Voronoi, Delaunay, and block-structured mesh refinement for solution of the shallow-water equations on the sphere. Mon. Wea. Rev., 137, 4208-4224.

Williamson, D. L., 1968: Integration of the barotropic vorticity equation on a spherical geodesic grid. Tellus, 20, 642-653.

, J. B. Drake, J. J. Hack, R. Jakob, and P. N. Swarztrauber, 1992: A standard test set for numerical approximations to the shallow water equations in spherical geometry. J. Comput. Phys., 102, 211-224. 